Dear Author,

Please, note that changes made to the HTML content will be added to the article before publication, but are not reflected in this PDF.

Note also that this file should not be used for submitting corrections. 
Research Article

\title{
Tuning cell adhesion by direct nanostructuring silicon into cell repulsive/adhesive patterns
}

\author{
Priyatha Premnath ${ }^{\mathrm{a}, 1}$, Amirhossein Tavangar ${ }^{\mathrm{a}, 1}$, Bo Tan ${ }^{\mathrm{b}}$, Krishnan Venkatakrishnan ${ }^{\mathrm{a}, *}$ \\ a Micro/Nanofabrication Laboratory, Department of Mechanical and Industrial Engineering, Ryerson University, 350 Victoria Street, Toronto, ON, Canada M5B 2K3 \\ ${ }^{\mathrm{b}}$ Nanocharacterization Laboratory, Department of Aerospace Engineering, Ryerson University, 350 Victoria Street, Toronto, ON, Canada M5B 2K3
}

\section{A R T I C L E I N F O}

Article history:

Received 3 May 2015

Received in revised form

16 July 2015

Accepted 26 July 2015

Keywords:

Silicon

Nanostructures

Cell guidance

Cytophilic/cytophobic patterns

HeLa

Superhydrophilicity

\begin{abstract}
A B S T R A C T
Developing platforms that allow tuning cell functionality through incorporating physical, chemical, or mechanical cues onto the material surfaces is one of the key challenges in research in the field of biomaterials. In this respect, various approaches have been proposed and numerous structures have been developed on a variety of materials. Most of these approaches, however, demand a multistep process or post-chemical treatment. Therefore, a simple approach would be desirable to develop bio-functionalized platforms for effectively modulating cell adhesion and consequently programming cell functionality without requiring any chemical or biological surface treatment. This study introduces a versatile yet simple laser approach to structure silicon (Si) chips into cytophobic/cytophilic patterns in order to modulate cell adhesion and proliferation. These patterns are fabricated on platforms through direct laser processing of Si substrates, which renders a desired computer-generated configuration into patterns. We investigate the morphology, chemistry, and wettability of the platform surfaces. Subsequently, we study the functionality of the fabricated platforms on modulating cervical cancer cells (HeLa) behaviour. The results from in vitro studies suggest that the nanostructures efficiently repel HeLa cells and drive them to migrate onto untreated sites. The study of the morphology of the cells reveals that cells evade the cytophobic area by bending and changing direction. Additionally, cell patterning, cell directionality, cell channelling, and cell trapping are achieved by developing different platforms with specific patterns. The flexibility and controllability of this approach to effectively structure Si substrates to cell-repulsive and cell-adhesive patterns offer perceptible outlook for developing bio-functionalized platforms for a variety of biomedical devices. Moreover, this approach could pave the way for developing anti-cancer platforms that are repellent to cancer cells but favourable for other types of cells.
\end{abstract}

(c) 2015 Published by Elsevier Inc.

\section{Introduction}

Cells are highly sensitive to the physical (e.g., nano-scaled topographical interaction), chemical (e.g., specific molecule or protein recognition), and mechanical (e.g., tissue-like stiffness) cues in their environment [1-3]. The main challenge in the field of biomaterials is using specific cues to develop functional platforms in order to modulate cell behaviour for a desired application.

Earlier studies in this field have pointed out that incorporating extracellular matrix (ECM) proteins or specific biomolecules onto or modifying the topography of biomaterial surfaces results in changes in cell morphologies, adhesion, proliferation, and migration [4]. Introducing micro- and nano-patterns onto biomaterial

\footnotetext{
* Corresponding author. Fax: +1 4169795056.

E-mail addresses: priyatha.premnath@ryerson.ca (P. Premnath), atavanga@ryerson.ca (A. Tavangar), tanbo@ryerson.ca (B. Tan), venkat@ryerson.ca (K. Venkatakrishnan).

${ }^{1}$ The first two authors made equal contributions.
}

surfaces using chemical and topographical cues has particularly been reported in numerous applications such as living cell biosensing [5], programmable apoptosis [6], cell isolation [7,8], cell patterning [9], and regenerative medicine [10,11]. Nonetheless, chemical modification of adhesive/anti-adhesive molecules on smooth surfaces may lead to the cross contamination of cells/ molecules and the migration of cells [2]. Moreover, unintentional altering the surface chemistry during the micro/nano structuring processes would cause the instability of chemical composition of the surfaces, which would eventually alter the cell phenotype [12].

In recent years, there has been a growing interest for controlled regulation of cellular systems by engineering topographical microand nano-features [4]. Advances in micro- and nano-technologies have provided required tools to precisely create and characterize substrates to successfully control a particular process (e.g., guide cell adhesion, regulate cell-biomaterial interaction) [1]. Such micro- and nano-substrates have been commonly fabricated in polydimethylsiloxane (PDMS) $[13,14]$, silicon $[9,15]$, titanium $[16,17]$, and polyacrylamide $[18,19]$, among which $\mathrm{Si}$ is widely 
used due to simplicity in the fabrication process, stiffness, nontoxicity, and degradation [20;21]. Although silicon micro- and nano-substrates benefit from a variety techniques, Si chip microfabrication, e.g., lithography and photolithography, has prevailed as the primary method to generate micro and nanostructured substrata to induce cellular alignment, known as contact guidance $[9,22,23]$. This method, however, demands elaborate, expensive setups and multistep processes [24].

One effective approach to fabricate micro-/nano-platforms for controlling cell function is ultrafast laser processing [25-27]. This technique has already been utilized by different research groups to alter the adhesion of cells on substrates by changing the wetting properties of Si surfaces $[25,28]$. The reported laser approaches, however, require vacuum settings and cannot be performed at atmospheric conditions. Moreover, they usually involve multiple steps including structuring the surfaces using laser processing in vacuum and then chemical treatment of the surfaces by coating the structures with various conformal layers, e.g., hydrophilic oxide layer or hydrophobic silane layer. These chemical treatments may adversely change the surface properties by introducing unknown toxins to the biological environment. Furthermore, various applications, such as tissue engineering, biosensing, cell sorting, biofouling modification, and micro devices, require platforms that have selective cell adhesiveness, for example, cytophilic and cytophobic patterns $[4,24,29,30]$. However, as the coating process is carried out in different media or/and through different methods, e.g., oxidation through thermal process or silanation through vapour adsorption from solution, generating different patterns (cytophilic/cytophobic) on the same platform is almost impossible. To this end, a versatile yet simple approach would be desirable in order to modulate cell adhesion through engineering cytophobic and cytophilic sites onto the same platforms, and to consequently program cell functionality without the need for additional chemical or biological surface treatments.

In this paper, we introduce a flexible single-step approach to develop platforms with cytophilic and cytophobic patterns using high-repetition femtosecond laser processing of Si. Laser processing of $\mathrm{Si}$, which is performed at ambient condition without the need for vacuum settings, simultaneously changes chemistry and topography of the surfaces and leads to the formation of cell-repulsive 3-D nanostructured zones, through a proper combination of change in surface topography and chemistry, without the requirement for post-chemical treatment. Since no post-chemical or -biological treatment is carried out on the surfaces, biocompatibility of the platforms are not affected; in other words, no unknown toxin, which may have adverse effects on biological environment, is introduced to the surfaces. The generated nanostructured patterns can be favourably tailored by changing certain laser parameters. We first developed several platforms on Si substrates using different laser-material interaction parameters. Then, we analyzed the morphology and physiochemical properties of the structures using scanning electron microscope (SEM), Energy-dispersive X-ray spectroscopy (EDX), X-ray powder diffraction (XRD), and contactangle measurement, respectively. We later investigated the ability of the developed platforms on programming HeLa cell behaviour. HeLa is one of the most common immortalized cell line that is used in laboratories around the world [31]. HeLa is particularly preferred for cell adhesion, spreading, and proliferation studies as they are able to evade apoptosis, which occurs due to lack of adhesion sites, primarily through multi-cellular aggregate formation [31-33]. The adhesion and morphology of the cells were investigated using SEM analysis and fluorescent microscopy. The versatility and simplicity of the proposed approach to generate patterning cytophobic sites in a single step without the use of post-chemical or -biological treatment will make it desirable for developing purpose-specific cellfunction regulators for numerous biomedical applications.

\section{Materials and methods}

\subsection{Nanostructured platform fabrication}

The fabrication of functionalized Si platforms was performed by megahertz $(\mathrm{MHz})$-repetition-rate ultrafast laser irradiation of undoped Si wafers at atmospheric condition. Si wafers (100) with a thickness of $500 \mu \mathrm{m}$ (University Wafers, USA) were diced into $1 \mathrm{~cm}^{2}$ chips. These chips were first cleaned with de-ionized (DI) water and ethanol and were rinsed off with DI water. The chips were then irradiated by a diode pumped, Yb-doped femtosecond laser system (Clark-MXR Inc. IM-PULSE Series Ultrashort Pulse laser) at laser pulse repetitions of $4,8,13$, and $26 \mathrm{MHz}$. The chips were mounted on a fixed stage and subjected to the incident laser beam with a computer-controlled high precision 2-D translation scanner. For the first part of the study, the Si platforms were fabricated by machining arrays of lines with separations of $100 \mu \mathrm{m}$ to $2 \mathrm{~mm}$ on the chips. Later, several platforms with varied patterns were created to control specific cell guidance. The power of the incident laser beam and the speed at which the laser beam scans the platforms were maintained at $10 \mathrm{~W}$ and $10 \mathrm{~mm} / \mathrm{s}$, respectively. Although several laser parameters, such as laser pulse width, peak power, pulse repetition rates, play an important role on the density of nanostructures generated on the platforms, the optimal parameters chosen for the study were a pulse width of $214 \mathrm{fs}$ and a laser pulse repetition rate of $26 \mathrm{MHz}$. All the laser parameters were computer-monitored in order to facilitate consistency and accuracy.

\subsection{Morphology and physicochemical characterization}

SEM (Hitachi S 5200) was used to characterize the surface morphology of the platforms. Individual nanoparticles forming 3-D nanostructures were studied using TEM (Hitachi H 700 CTEM). In order to detach the nanoparticle networks from the substrates for TEM analysis, the samples were dipped in isopropanol solution and then ultrasonically vibrated. A drop from this solution was then placed on a copper mesh and allowed to dry in a desiccator. EDX (Hitachi S 5200) was carried out to determine the elemental composition of the surfaces of the platforms. Phase analysis of the platforms surfaces was done using XRD.

\subsection{Contact angle measurement}

Contact angle measurements (OCA 35) were carried out using the sessile drop method. A $2 \mu \mathrm{l}$ distilled water droplet was applied on the platforms surfaces by a micro-syringe. The image of each droplet on the surface was recorded using a digital microscope, and the images were then used to automatically measure the angle formed at the liquid-solid interface using SCA 20 software (Neurtek). The mean value of the contact angle measurements was calculated from three individual measurements that were taken at different locations on the platforms.

\subsection{Cell culture}

Cervical cancer cells (HeLa-Henrietta Lacks) $\left(\right.$ ATCC $^{\circledR}$ CCL-2, USA) were used to study the bio-functionality of the fabricated platforms. The cells were grown in Dulbecco's Modified Eagle's Medium (DMEM/F12) supplemented with 10\% fetal bovine serum (FBS) and $1 \%$ pen-strep, which was incubated at $37{ }^{\circ} \mathrm{C}$ under a humidified $5 \% \mathrm{CO}_{2}-95 \%$ air atmosphere. Prior to cell interaction, the Si platforms were sterilized with ethanol and DI water and kept under UV light for $20 \mathrm{~min}$. Next, the platforms were placed in petri dishes containing $3 \mathrm{ml}$ of DMEM/F12 medium and 10\% FBS per dish, and HeLa cells were seeded at a density of $10^{5}$ cells $/ \mathrm{ml}$. 
The petri dishes were then placed in an incubator for 24 and $48 \mathrm{~h}$.

The morphologies of HeLa cells seeded on the platforms were studied using SEM. After the incubation period, the platforms were fixed in $2 \%$ glutaraldehyde for $1 \mathrm{~h}$. They were then immersed in $0.1 \mathrm{M}$ sodium cacodylate buffer with $0.2 \mathrm{M}$ sucrose at $\mathrm{pH} 7.3$ for 20 min. Afterwards, dehydration of the cells was done through increasing concentrations of ethanol (from 10\% to 100\%) for $20 \mathrm{~min}$ each. The platforms were later critical-point dried on $100 \%$ ethanol. The SEM analysis was conducted at an acceleration voltage of $5 \mathrm{kV}$ and magnifications that were varied between 100 and 10,000 times.

Cell viability on the Si platforms was measured using CalceinAM Alexa Fluor ${ }^{\circledR} 488$ (Life Technologies, Catalogue number: L-3224) and 4',6-Diamidino-2-Phenylindole, Dihydrochloride (DAPI-Life Technologies, Catalogue number: D1306) to stain cell cytoskeleton and nucleus, respectively. After incubation period, the platforms were first fixed in methanol free $4 \%$ paraformaldehyde in PBS and then immersed in a solution of 5\% non-fat milk with $0.1 \%$ triton and PBS before incubation in order to prevent non-specific binding and permeabilization of cells, respectively [34-38] The platforms were then incubated for $15 \mathrm{~min}$ at $37^{\circ} \mathrm{C}$ and finally were observed under a fluorescence microscope (Nikon).

\section{a}

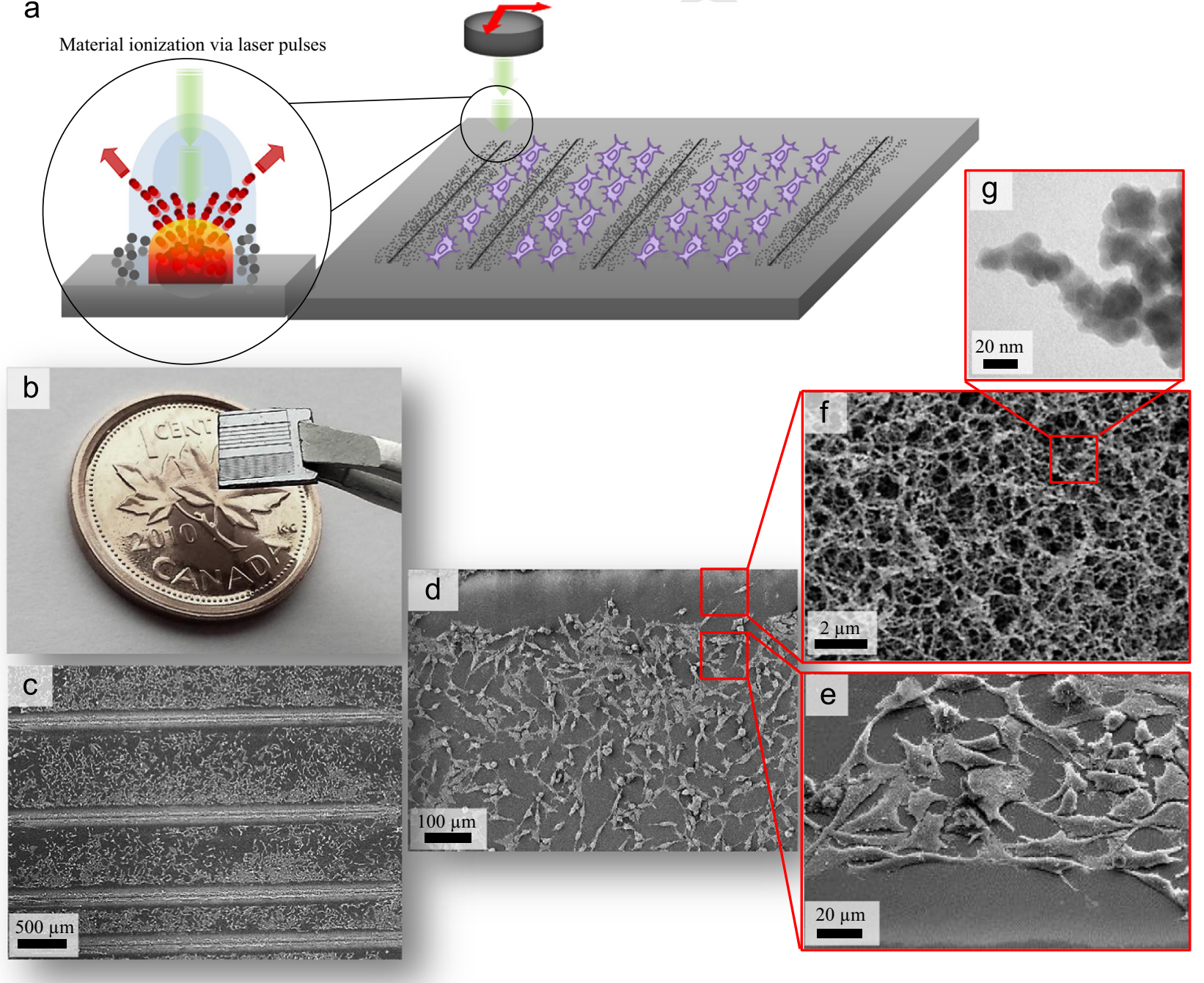

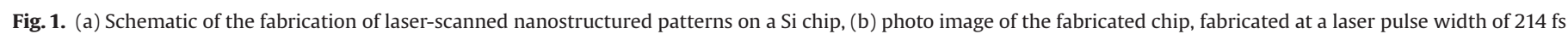

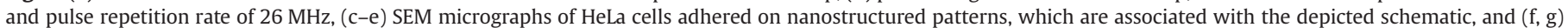
SEM and TEM micrographs of the 3-D nanostructures.

\section{Results}

\subsection{Fabrication and characterization of Si platforms}

The bio-functionalized Si platforms were fabricated by femtosecond laser processing of silicon chips at ambient condition. Femtosecond laser pulses heat materials to higher pressure and pulse lasers with comparable laser incident fluences, i.e., pulse energy per laser beam area, because the energy is distributed to the material before any significant thermal conduction occurs in it $[39,40]$. Also, laser processing at $\mathrm{MHz}$ pulse repetition rates allows successive laser pulses to irradiate the same spot on the target surface. As a result, a continuous flow of the plume vapour, which contains vaporized nano-species of the material, is maintained for longer period of time. That leads to the growth of nanoparticles, which will then aggregate into 3-D nanostructures after further collision, as shown in the schematic in Fig. 1. Therefore, femtosecond laser pulses with $\mathrm{MHz}$ repetition rates are required to fabricate such interwoven nanostructures on the platforms.

The computer-controlled laser scanner allows for precise $\mathrm{X}-\mathrm{Y}$ translational movement of the laser beam on the substrate surface. As the laser beam scans the substrate, it ablates its surface into temperature in a shorter time interval compared to those of longer- 
desired nanostructured patterns, which have already been created in the computer. The density of the fabricated nanostructures can be controlled by several laser parameters such as laser pulse width and repetition rate. In this study, we fabricated platforms that were laser-patterned at pulse width of 214,771 , and $1428 \mathrm{fs}$ and laser repetition rates of $4,8,12$, and $26 \mathrm{MHz}$ and studied their functionality on cell adhesion and proliferation. The preliminary results (data not shown) from our study suggested that the best functionality on programming HeLa cell adhesion and proliferation was achieved at a laser pulse width of $214 \mathrm{fs}$ and pulse repetition rate of $26 \mathrm{MHz}$. Thus, we only presented the results of the platforms fabricated at these parameters. Fig. 1 shows a schematic of the fabrication process of laser-scanned nanostructured patterns on a Si chip and how it guides cell adhesion toward desired cell arrangement and directionality (Fig. 1a), the photo image of the fabricated chip fabricated at a laser pulse width of $214 \mathrm{fs}$ and pulse repetition rate of $26 \mathrm{MHz}$ (Fig. 1b), the SEM micrographs of HeLa cells adhered on nanostructured patterns associated with the depicted schematic (Fig. 1(c-e)), and the close-up SEM and TEM micrographs of the 3-D nanostructures (Fig. 1(f and g)).

One of the advantages of the proposed approach in this study is that the laser processing is performed at ambient atmosphere. This eliminates the need for an ambient gas or vacuum chamber. Also, laser interaction with $\mathrm{Si}$ at ambient atmosphere results in oxidation of the fabricated nanostructures, which leads to formation of silica nanostructures. Hence, the changes in both topography, resulted from 3-D nanostructures, and chemistry, due to the oxidation of the surface, would provide proper cues to influence programmable cell adhesion and proliferation.

The chemistry and crystal structure of the nanostructured patterns were evaluated using EDX and XRD analyses, respectively. As shown in Fig. 2a, EDX analysis of the nanostructured patterns indicated the presence of oxygen and silicon. Fig. $2 \mathrm{~b}$ compares the XRD patterns of an untreated $\mathrm{Si}$ substrate and the nanostructures processed on a Si chip. XRD patterns of the nanostructures confirmed the presence of crystalline silicon in (111), (220) and (311) orientations as well as amorphous silicon oxides. It is observed that the nanostructures are composed of randomly oriented micro crystallites of silicon and amorphous silicon oxides. This arbitrary structure could be associated with varying plasma plume mechanisms and collisions of species with different crystalline structures [41].

In biological milieu, the chemistry and wettability of surfaces play important roles in meditating protein adsorption and cell adhesion $[42,43]$. Thus, wettability of the treated areas, which consisted of silica nanostructures, and untreated $\mathrm{Si}$ areas on the platforms was studied using sessile drop contact angle measurement of a distilled water droplet. As illustrated in Fig. 3, a contact angle $<3^{\circ}$ was observed during the contact angle measurements of the treated areas, which was opposed to that of untreated $\mathrm{Si}$ $\left(65^{\circ} \pm 1\right)$. Complete spreading of the water droplet on the treated areas suggested that structuring $\mathrm{Si}$ into 3-D nanostructured silica made it superhydrophilic, which was the result of changes in both chemistry and topography of the treated areas.

\subsection{Cell interaction with the platforms}

In the next step, cell adhesion and viability studies were performed using HeLa cells in order to examine the functionality of the fabricated platforms on modulating cellular behaviours. SEM micrographs of HeLa cells seeded on the Si platforms exhibited different cell concentration and morphology on untreated and treated areas. As illustrated in Fig. 4, the cells proliferated and adhered well to the surface on the untreated Si area. On the other hand, the treated areas on the platforms presented different cell functionality. The number of adhered cells per unit area decreased dramatically on the treated area, consisting of nanostructures. Moreover, the SEM micrographs of HeLa cells cultured on the platforms for $24 \mathrm{~h}$ and $48 \mathrm{~h}$, shown in Fig. 4, revealed that cells appeared to be repelled by the nanostructured areas and forced to migrate and accumulate on untreated zones in order to evade unfavourable contacts with the nanostructured areas.

The morphology of cells growing on surfaces can qualitatively provide information about the cell-surface interaction [25]. Large polygonal cell spreading is a strong indication of intimate interaction between the cells and the surface. In contrast, premature filopodial extension, decreased number of filopodia, cell clustering, and small round shape imply poor cell-surface interaction $[10,25,27,44]$. From SEM micrographs presented in Fig. 5(a-c), it can be seen that the cells growing in the vicinity of the nanostructured areas appeared to be smaller and their side facing the
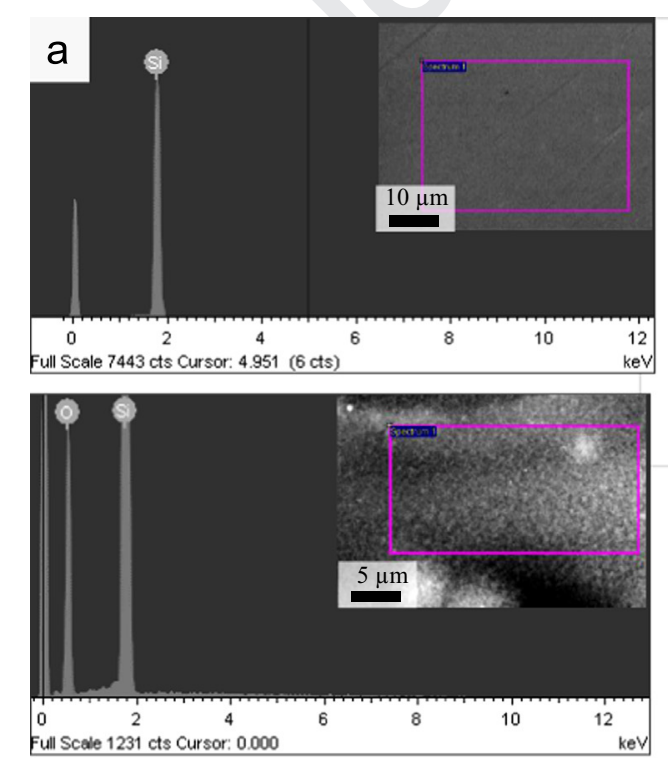

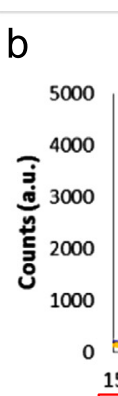

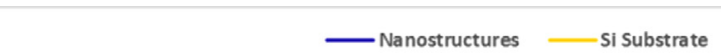
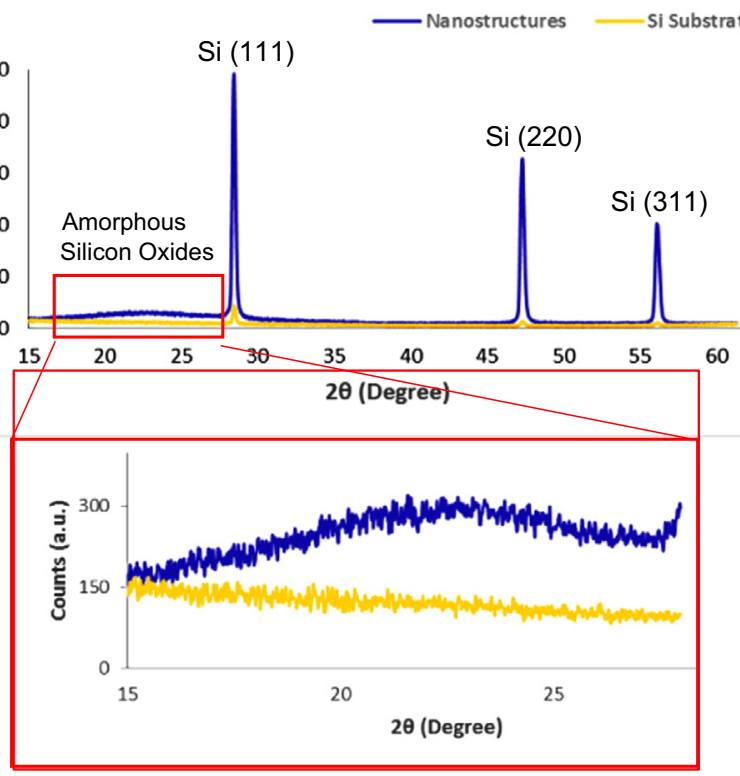

Fig. 2. EDX analysis of the surfaces of untreated Si substrate ((a) top) and fabricated nanostructures ((a) bottom). (b) XRD patterns of untreated Si substrate and nanoslaser processing in ambient air. 

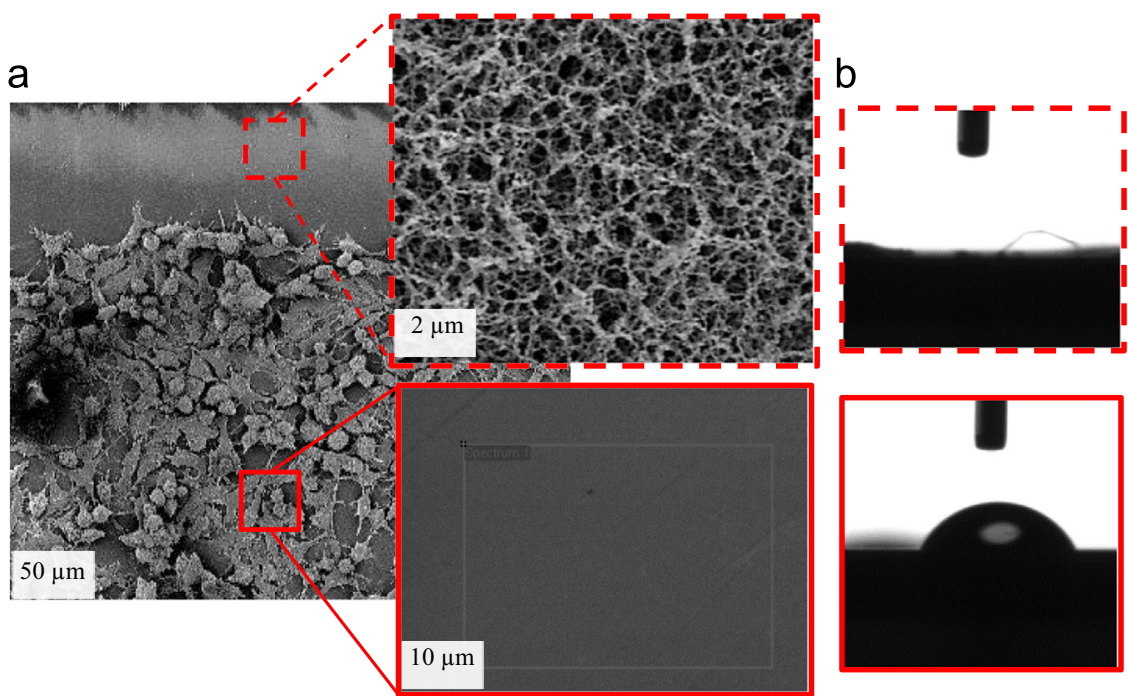

C

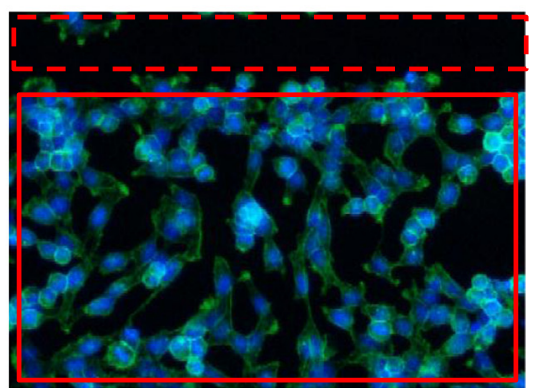

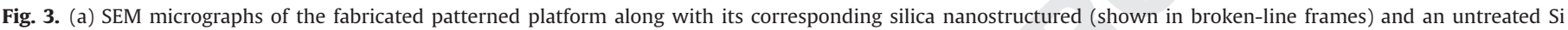

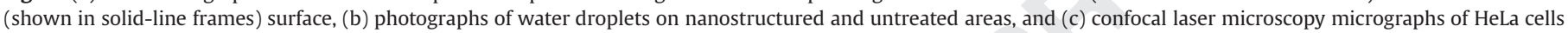
cultured for $24 \mathrm{~h}$ on the platforms. nanostructures developed no or relatively reduced number of filopodial extensions and adhesion points compared to their other side facing untreated Si. Even if any filopodium was extended toward the nanostructured areas, it appeared to be detached after adhesion, as observed for several cells grown on the superhydrophilic areas (Fig. 5(c)). Although a few number of cells adhered on the treated area on some parts, there was a distinctive difference in their morphology. The cells that adhered to superhydrophilic nanostructured areas formed rounded-shape phenotype, which indicates poor adhesion to the surface (Fig. 5(d-f)). This type of cell morphology could therefore be used to assess the quality of cell-surface interactions.

In order to further investigate the functionality of the nanostructured patterns for different biomedical applications, e.g., cell trapping, cell challenging, cell directionality, several platforms with different nanostructured configurations were fabricated. As observed in the SEM micrographs shown in Fig. 6(a-e), cell repelling, cell directionality, cell channelling, and cell trapping were achieved. In addition, migration of cells along channels with different widths were accomplished (Fig. 6. (f-h)). This property is one of the requirements for lab-on-a-chip and other microfluidic devices. Trapping or isolating a single cell or a number of cells, also, is desirable in various biomedical applications such as drug testing, fundamental biological studies, and toxicology. The findings from this study suggest that the proposed approach simply and effectively enables fabricating areas for cell isolating purposes [24].

Cell viability of the platforms was evaluated using HeLa cells and a cell viability assay kit in which the actin cytoskeleton and the nucleus of cells were stained in green and blue, respectively. The results from cell viability tests were consistent with previous SEM observation of cell morphology and adhesion patterns. As shown in Fig. 7, cell spreading and adhesion were hindered on superhydrophobic nanostructured areas. This along with cell acpatterns together with cells bending around these areas suggested the cytophobicity and repelling properties of the nanostructured patterns. By choosing proper laser processing parameters and appropriate patterns, the shape and width of these cytophobic sites can be perfectly controlled in order to guide cells toward a desired configuration and functionality.

\section{Discussion}

Cell response to the surface that they come in contact with mostly depends on the surface topography, chemistry, or the combination of both, the surface energy [25]. Therefore, research in biomaterial study has focused on developing platforms with suitable chemical or/and topographical cues in order to tune cumulation on the untreated areas between the nanostructured
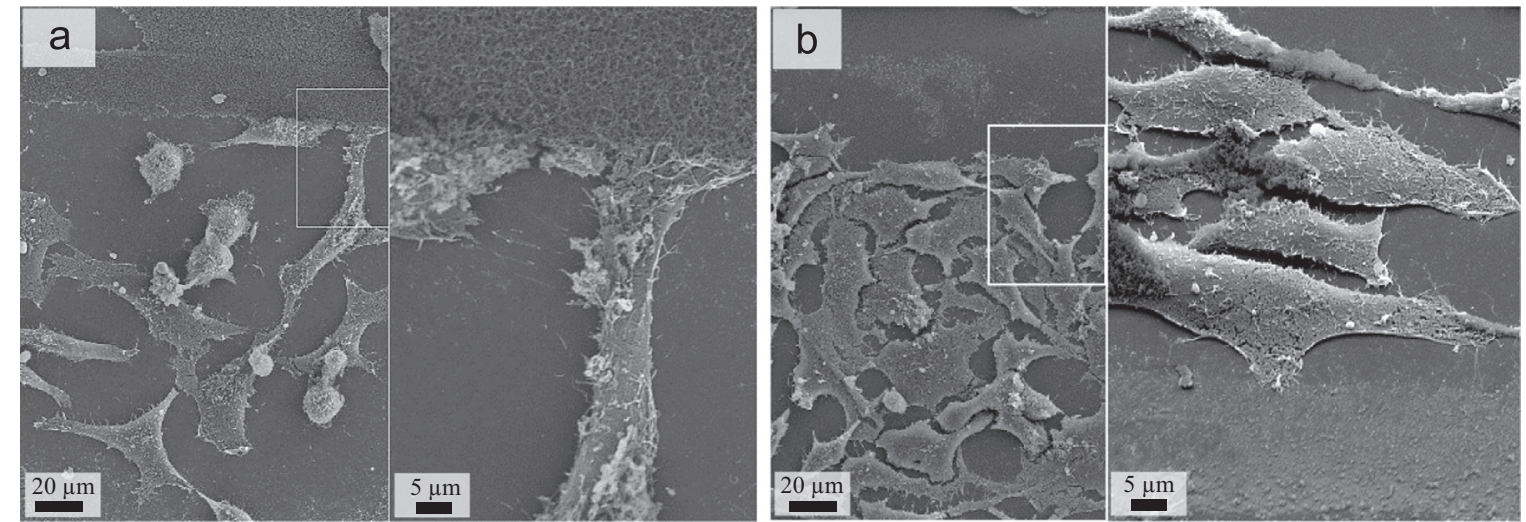

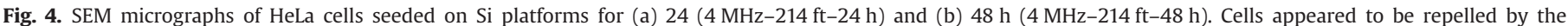
nanostructured areas and forced to migrate and accumulate on untreated zones to avoid unfavourable contacts with the nanostructured areas. 

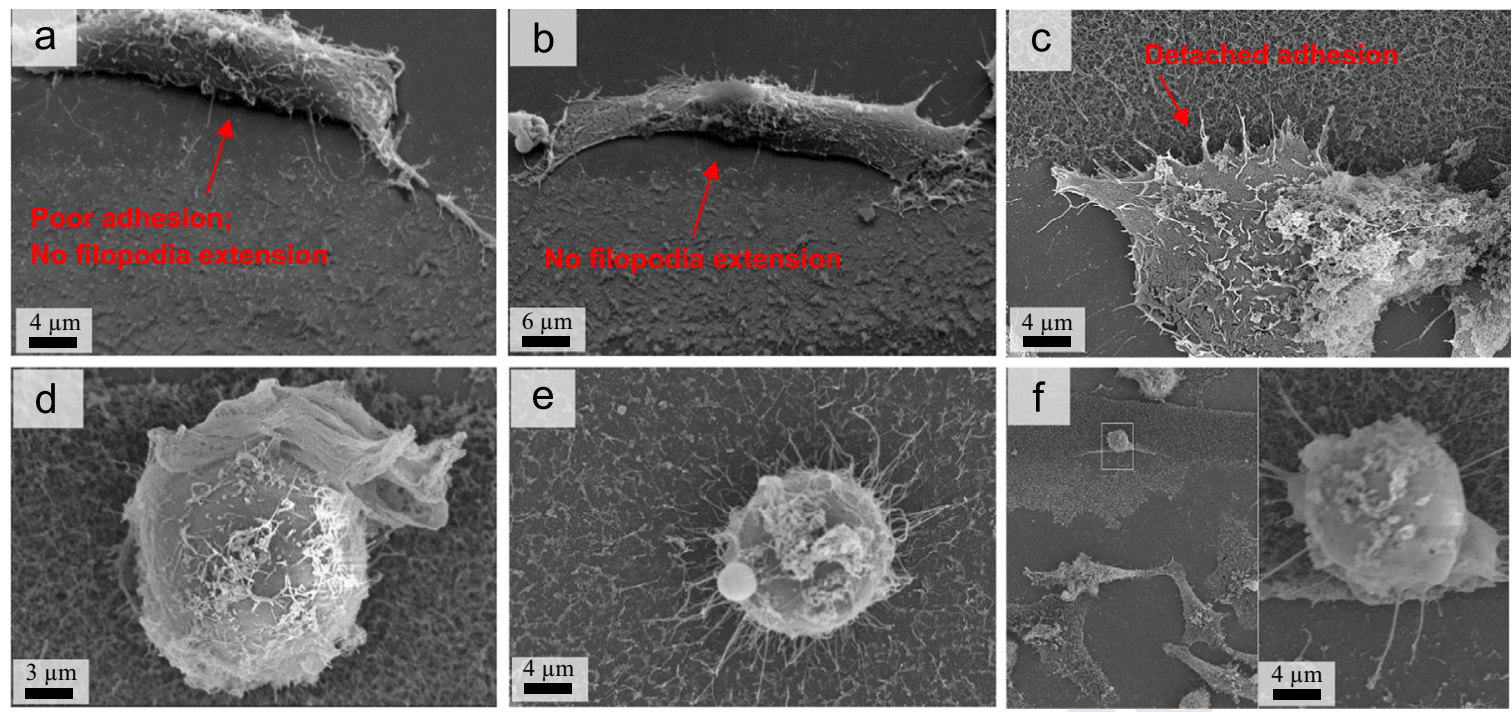

Fig. 5. SEM micrographs of HeLa cells adhered on vicinity $(a-c)$ and on $(d-f)$ nanostructured areas.

desirable cell function for a specific biomedical application. For example, long term stability of cardiovascular stents in the body highly depends on the enhanced cell controllability on their surfaces, which requires particular augmentation or prevention of smooth and epithelial cell growth [45]. Also, in order to mimic the growth of neural cells in vitro, directed cell growth is a priority, which necessitates the need for functionalized platforms to control directed growth, migration and adhesion of the cells [46]. Furthermore, biomedical devices such as biosensors demand high level of precision regarding the cell growth. Micro-/nano-diagnostic devices also require directed cell flow within microfluidic channels. Moreover, certain applications necessitate singular cell isolation for investigation, e.g., for investigating the prompt cell response to reagents, for tracking the molecular state of stem cells $[47,48]$. Therefore, a versatile yet simple approach would be desirable in order to develop bio-functionalized platforms for effectively modulating cell adhesion and consequently programming cell functionality.

In this study, bio-functionalized Si platforms were developed by laser processing of Si chips at ambient atmosphere. As the laser beam scans a chip, it ablates the surface and translates the desired computer-generated configuration into nanostructured patterns. The ablation of Si substrates in ambient air leads to a high temperature reactive plasma plume consisting $\mathrm{Si}$ ions in which the presence of any trace of reactive gases, such as oxygen in case of ambient air, will results in chemical reactions. The evaporated $\mathrm{Si}$ ions react with the surrounding oxygen in the frontier of the expanding plume, and thus $\mathrm{Si}-\mathrm{O}$ molecular monomers are formed. Since the oxidation of $\mathrm{Si}$ to $\mathrm{SiO}_{2}$ is highly exothermic, formation of $\mathrm{SiO}_{2}$ molecules would be more likely. These molecules then collide as a result of thermal motion and flow to the outer areas of the plume where temperature is lower. This will later cause molecular supersaturation, which consequently leads to nucleation. After the temperature drops further below the boiling or sublimation point, silica nanostructures start to form. This is consistent with the results from EDX and XRD analyses, which confirmed the presence of oxygen and amorphous silica phases in the nanostructures, respectively. Therefore, our results suggested that both the changes in surface topography, resulted from 3-D nanostructures, together with changes in the surface chemistry, due to the oxidation of
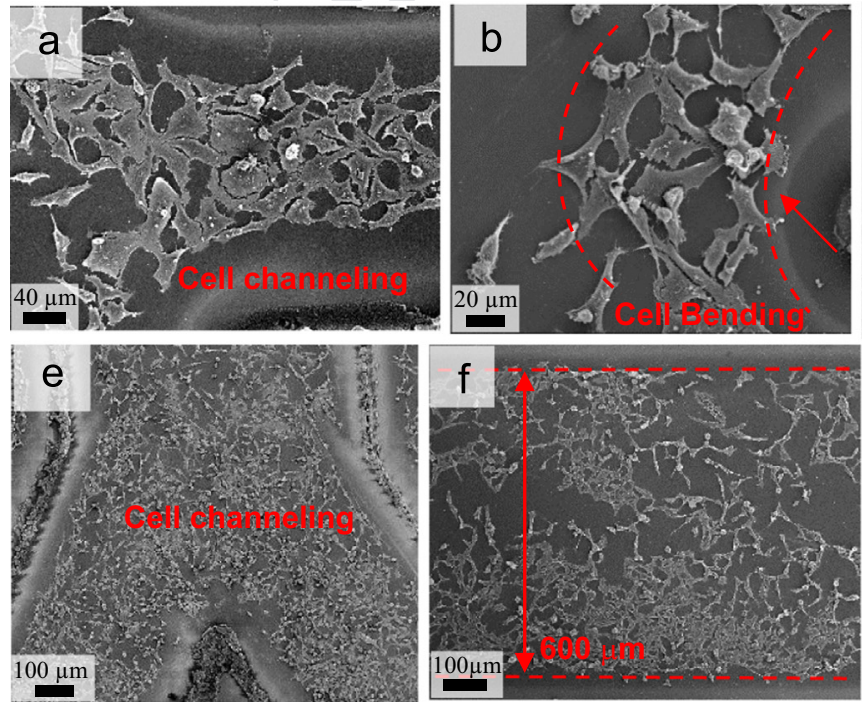
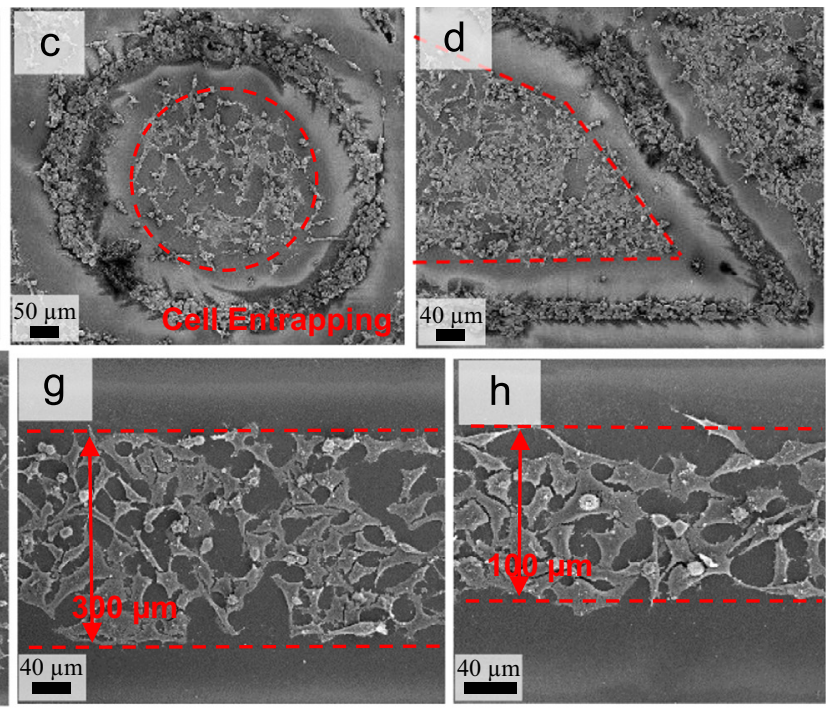

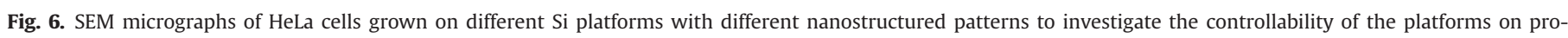

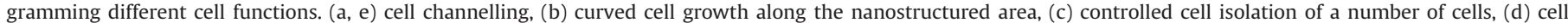
directionality along the desired pattern, and $(\mathrm{f}-\mathrm{h})$ controlled cell migration along channels with different widths. 

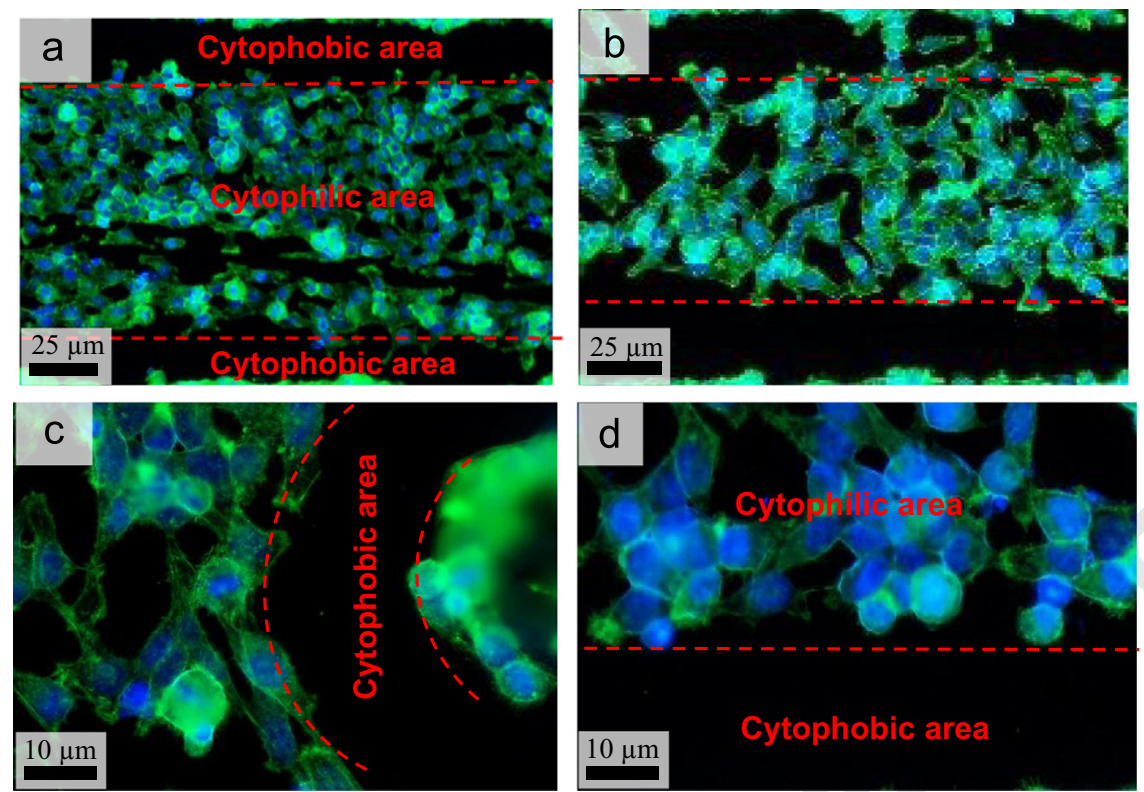

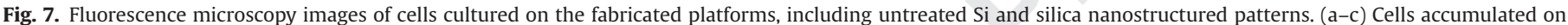

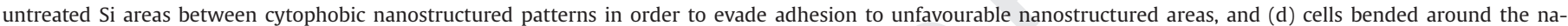
nostructure area as they were repelled by the cytophobic areas.

surface, provided signals to stimulate tuneable cell adhesion and proliferation. The results from contact angle measurement showed that such modifications in topography and chemistry made the surface superhydrophilic. This is in accordance with previous studies that have indicated that both surface oxidation and nanotopography contribute to superhydrophilicity of surfaces $[49,50]$.

Our studies on HeLa cell interaction with the fabricated platforms showed that superhydrophilic nanostructures effectively prevented the cells from adhering to the surfaces and forced them to migrate onto the untreated areas. This cytophobicity behaviour could be explained by the surface wettability and surface chemistry of the nanostructures. Although cell adhesion mainly linked to the surface wettability, it is also influenced by the surface chemistry and the type of cells interacting with the surfaces $[33,42,43]$. Since the surface chemistry directly correlates to the surface protein adsorption, cell adhesion is directly associated with the ability of a surface to adsorb proteins.

A biomaterial coming into contact with the biological milieu rapidly adsorbs proteins in the medium to its surface before any cell adhesion occurs. The surface properties, therefore, govern the type, amount, and arrangement of adsorbed proteins (small protein such as albumin). This eventually controls subsequent protein exchange processes to adhesive proteins (larger proteins such as fibronectin and vitronectin) and cell adhesion to the surface $[31,51,52]$. In case of in vitro studies, upon exposing to the culture medium supplemented with FBS, the biomaterial surface promptly adsorbs the proteins in the serum before any cell attaches to it [33]. The kind of adsorbed protein, however, is very important for subsequent cell adhesion behaviour. It is known that cell adhesive serum proteins, i.e., fibronectin and vitronectin, significantly contribute to the cell adhesion to the surface of biomaterials [33]. Nonetheless, albumin, which is also the most abundant serum protein $(35-50 \mathrm{mg} / \mathrm{mL}$ ), is favourably adsorbed onto the surfaces during early stage of biomaterial-serum contact. The adsorbed albumin is later expected to be substituted by cell adhesive proteins, which is highly dependant to the surface properties $[33,53]$.

Previous literature has shown that hydrophobic surfaces strongly adsorb albumin to the degree that it cannot then be displaced by cell adhesive proteins $[31,33]$. Also, these surfaces have been reported to promote protein denaturation. On the other hand, moderately hydrophilic surfaces are thought to promote adsorption of cell adhesive proteins and consequently cell adhesion $[3,31]$. On superhydrophilic surfaces, however, protein adsorption is the lowest [31]. Even if there is any cell adsorption, these surfaces are believed to hold cell-adhesive proteins with relatively weak forces. The weak protein-surface bond will lead to the detachment of such proteins from the surfaces particularly at later culture stages when they have attached to cells [3].

With respect to the surface chemistry, oxygen-terminated and $\mathrm{OH}$ surfaces are known to repulse protein adsorption due to tightly bound water and the subsequent strong hydration forces $[31,49,54]$. In regards to surface topography, prior studies have reported that nanostructures may promote cell adhesion of one type of cell but at the same time may discourage another. Several studies have previously reported a decline in cell adhesion, proliferation, and migration on nanostructured surfaces $[20,55]$. For instance, superhydrophilic oxygen-terminated nanostructured diamond surfaces almost completely repelled human mesenchymal stem cells. On the contrary, moderately hydrophilic nano-diamond surfaces supported the growth, adhesion, and spreading of these cells [56]. In other studies, extremely hydrophilic surfaces also discouraged the adhesion of PC12 cell attachment [57;21], though, HLE cells were found to adhere to such surfaces to a certain extent [21].

The abovementioned findings from other studies are in accordance with our results indicating that laser-rendering of $\mathrm{Si}$ substrate to oxygen-terminated nanostructured silica leads to superhydrophilicity of the surfaces and changes in the surface chemistry. These properties turn the surface from adhesive to repulsive for HeLa cells, which is consistent with the results reported for the same cell line on extremely hydrophilic surfaces [33].

As mentioned before, cell adhesion is not only controlled by the surface properties of biomaterials but also is influenced by the type of cells interacting with the surfaces. Thus, a surface that is adhesive for a type of cell might be repulsive for another cell line. For example, it has been shown that HeLa cells are more sensitive to surface properties than fibroblasts (a model for stromal cells which can be found in matrix and connective tissues) [31]. Moreover, fibroblasts are known to attach well on superhydrophilic surfaces $[10,27,58]$. This could particularly be an advantage where there is a need for platforms that encourage 
adhesion of one type of cell (e.g., fibroblast) and discourage another (e.g., cancer cells such as HeLa). Further studies will be useful to understand the bio-functionality of these patterned platforms with different cell lines. This capability to selectively tailor the surface properties of desired patterns on Si platform in order to make it repulsive to one type of cell and adhesive to another would allow manipulation of the cell behaviour for a desired application.

\section{Conclusion}

In summary, a flexible yet simple laser approach was introduced to develop Si platforms for modulating cell behaviour through cytophobic and cytophilic patterns. These patterns were created on platforms through laser scanning and ablating Si substrates, which translated a desired computer-generated configuration into patterns. It was found that direct laser scanning of Si introduced randomly oriented crystalline $\mathrm{Si}$ and amorphous oxidized 3-D nanostructures with high surface energy and superhydrophilicity. The results suggested that the fabricated nanostructured patterns and their surface properties could effectively be tailored by altering certain laser parameters. The functionality of fabricated platforms was investigated using HeLa cells. The findings from in vitro studies revealed that the nanostructured patterns almost completely repelled HeLa cells from adhesion and forced them to migrate to untreated Si areas. It was discussed that this behaviour was attributed to both surface energy and wettability of the nanostructures. It was also revealed from SEM analysis of the morphology of the cells that the cells grown in the vicinity of the nanostructured patterns did not develop filopodia on the side facing the nanostructures and completely evaded the cytophobic area by bending and changing direction. Further, cell patterning, cell directionality, cell channelling, and cell trapping were achieved by introducing specific patterns to the Si platforms. It was finally argued that since cell adhesion was also governed by the type of cells interacting with the surfaces, the nanostructured areas, which is repulsive to cancer cells, might be adhesive for a type of cell such as fibroblast, which is less sensitive to surface physiochemical properties. Therefore, the proposed approach would pave the way to develop anti-cancer platforms that are repellent to cancer cells but favourable for other types of cells. Besides, the simplicity and controllability of this approach to rapidly structure Si chips to cell-repulsive and cell-adhesive patterns offer perceptible outlook for developing bio-functionalized platforms for a verity of biomedical devices such as biosensors, lab-ona chip, and implantable devices.

\section{Acknowledgement}

This research was funded by Natural Science and Engineering Research Council of Canada (NSERC).

\section{References}

[1] C.J. Bettinger, R. Langer, J.T. Borenstein, Engineering substrate topography at the micro- and nanoscale to control cell function, Angew. Chem. Int. Ed. 48 (30) (2009) 5406-5415.

[2] X. Liu, S. Wang, Three-dimensional nano-biointerface as a new platform for guiding cell fate, Chem. Soc. Rev. 43 (8) (2014) 2385-2401.

[3] L. Bacakova, E. Filova, M. Parizek, T. Ruml, V. Svorcik, Modulation of cell adhesion, proliferation and differentiation on materials designed for body implants, Biotechnol. Adv. 29 (6) (2011) 739-767.

[4] J.Y. Lim, H.J. Donahue, Cell sensing and response to micro- and nanostructured surfaces produced by chemical and topographic patterning, Tissue Eng. 13 (8) (2007) 1879-1891.
[5] V. Chabot, C.M. Cuerrier, E. Escher, V. Aimez, M. Grandbois, P.G. Charette, Biosensing based on surface plasmon resonance and living cells, Biosens. Bioelectron. 24 (6) (2009) 1667-1673.

[6] L.B. Frankel, N.R. Christoffersen, A. Jacobsen, M. Lindow, A. Krogh, A.H. Lund, Programmed cell death 4 (PDCD4) is an important functional target of the MicroRNA miR-21 in breast cancer cells, J. Biol. Chem. 283 (2) (2008) 1026-1033.

[7] S. Wang, K. Liu, J. Liu, Z.T.-F. Yu, X. Xu, L. Zhao, T. Lee, E.K. Lee, J. Reiss, Y.-K. Lee, L.W.K. Chung, J. Huang, M. Rettig, D. Seligson, K.N. Duraiswamy, C.K.-F. Shen, H.R. Tseng, Highly efficient capture of circulating tumor cells using nanostructured silicon substrates with integrated chaotic micromixers, Angew. Chem. Int. Ed. Engl. 50 (13) (2011) 3084-3088.

[8] A.A. Adams, P.I. Okagbare, J. Feng, M.L. Hupert, D. Patterson, J. Göttert, R. L. McCarley, D. Nikitopoulos, M.C. Murphy, S.A. Soper, Highly efficient circulating tumor cell isolation from whole blood and label-free enumeration using polymer-based microfluidics with an integrated conductivity sensor, J. Am. Chem. Soc. 130 (27) (2008) 8633-8641.

[9] C.P. Unsworth, H. Holloway, E. Delivopoulos, A.F. Murray, M.C. Simpson, M. E. Dickinson, E.S. Graham, Patterning and detailed study of human hNT astrocytes on parylene-C/silicon dioxide substrates to the single cell level, Biomaterials 32 (27) (2011) 6541-6550.

[10] A. Tavangar, B. Tan, K. Venkatakrishnan, The influence of laser-induced 3-D titania nanofibrous platforms on cell behavior, J. Biomed. Nanotechnol. 9 (11) (2013) 1837-1846.

[11] K. Tuzlakoglu, N. Bolgen, A.J. Salgado, M.E. Gomes, E. Piskin, R.L. Reis, Nanoand micro-fiber combined scaffolds: a new architecture for bone tissue engineering, J. Mater. Sci. Mater. Med. 16 (12) (2005) 1099-1104.

[12] M.M. Stevens, J.H. George, Exploring and engineering the cell surface interface, Science 310 (5751) (2005) 1135-1138.

[13] L. Wang, S.K. Murthy, W.H. Fowle, G.A. Barabino, R.L. Carrier, Influence of micro-well biomimetic topography on intestinal epithelial Caco-2 cell phenotype, Biomaterials 30 (36) (2009) 6825-6834.

[14] J. Mai, C. Sun, S. Li, X. Zhang, A microfabricated platform probing cytoskeleton dynamics using multidirectional topographical cues, Biomed. Microdevices 9 (4) (2007) 523-531.

[15] M. Nikkhah, J.S. Strobl, V. Srinivasaraghavan, M. Agah, Isotropically etched silicon microarrays for rapid breast cancer cell capture, Sens. J. IEEE 13 (3) (2013) 1125-1132.

[16] S. Oh, K.S. Brammer, Y.S.J. Li, D. Teng, A.J. Engler, S. Chien, S. Jin, Stem cell fate dictated solely by altered nanotube dimension, Proc. Natl. Acad. Sci. 106 (7) (2009) 2130-2135

[17] L. Zhao, S. Mei, P.K. Chu, Y. Zhang, Z. Wu, The influence of hierarchical hybrid micro/nano-textured titanium surface with titania nanotubes on osteoblast functions, Biomaterials 31 (19) (2010) 5072-5082.

[18] X. Tang, M.Y. Ali, M.T.A. Saif, A novel technique for micro-patterning proteins and cells on polyacrylamide gels, Soft Matter 8 (27) (2012) 7197-7206.

[19] D. Lü, C. Luo, C. Zhang, Z. Li, M. Long, Differential regulation of morphology and stemness of mouse embryonic stem cells by substrate stiffness and topography, Biomaterials 35 (13) (2014) 3945-3955.

[20] M.J. Dalby, M.O. Riehle, D.S. Sutherland, H. Agheli, A.S.G. Curtis, Changes in fibroblast morphology in response to nano-columns produced by colloidal lithography, Biomaterials 25 (23) (2004) 5415-5422.

[21] S.P. Low, K.A. Williams, L.T. Canham, N.H. Voelcker, Evaluation of mammalian cell adhesion on surface-modified porous silicon, Biomaterials 27 (26) (2006) 4538-4546.

[22] D. Zahor, A. Radko, R. Vago, L.A. Gheber, Organization of mesenchymal stem cells is controlled by micropatterned silicon substrates, Mater. Sci. Eng. C 27 (1) (2007) 117-121.

[23] A.I. Teixeira, G.A. Abrams, P.J. Bertics, C.J. Murphy, P.F. Nealey, Epithelial contact guidance on well-defined micro- and nanostructured substrates, J. Cell Sci. 116 (10) (2003) 1881-1892.

[24] P. Premnath, B. Tan, K. Venkatakrishnan, Programming cell fate on bio-functionalized silicon, Colloids Surf. B Biointerfaces 128 (2015) 100-105.

[25] A. Ranella, M. Barberoglou, S. Bakogianni, C. Fotakis, E. Stratakis, Tuning cell adhesion by controlling the roughness and wettability of 3D micro/nano silicon structures, Acta Biomater. 6 (7) (2010) 2711-2720.

[26] V. Zorba, L. Persano, D. Pisignano, A. Athanassiou, E. Stratakis, R. Cingolani, P. Tzanetakis, C. Fotakis, Making silicon hydrophobic: wettability control by two-lengthscale simultaneous patterning with femtosecond laser irradiation, Nanotechnology 17 (13) (2006) 3234.

[27] P. Premnath, B. Tan, K. Venkatakrishnan, Direct patterning of free standing three dimensional silicon nanofibrous network to facilitate multi-dimensional growth of fibroblasts and osteoblasts, J. Biomed. Nanotechnol. 9 (11) (2013) 1875-1881.

[28] D. Rajput, S.W. Crowder, L. Hofmeister, L. Costa, H.-J. Sung, W. Hofmeister, Cell interaction study method using novel 3D silica nanoneedle gradient arrays, Colloids Surf. B Biointerfaces 102 (2013) 111-116.

[29] M. Goto, K. Sato, A. Murakami, M. Tokeshi, T. Kitamori, Development of a microchip-based bioassay system using cultured cells, Anal. Chem. 77 (7) (2005) 2125-2131.

[30] Y. Wang, Z. Xu, L.C. Kam, P. Shi, Site-specific differentiation of neural stem cell regulated by micropatterned multicomponent interfaces, Adv. Healthc. Mater. 3 (2) (2014) 214-220.

[31] P.A. Santos, C.S. Rocha, M.S. Baptista, Adhesion and proliferation of HeLa and fibroblast cells on chemically-modified gold surfaces, Colloids Surf. B Biointerfaces 123 (2014) 429-438. 
[32] X. Zhong, F.J. Rescorla, Cell surface adhesion molecules and adhesion-initiated signaling: understanding of anoikis resistance mechanisms and therapeutic opportunities, Cell. Signal. 24 (2) (2012) 393-401.

[33] Y. Arima, H. Iwata, Effect of wettability and surface functional groups on protein adsorption and cell adhesion using well-defined mixed self-assembled monolayers, Biomaterials 28 (20) (2007) 3074-3082.

[34] V.T. Lee, D.M. Anderson, O. Schneewind, Targeting of Yersinia Yop proteins into the cytosol of HeLa cells: one-step translocation of YopE across bacterial and eukaryotic membranes is dependent on SycE chaperone, Mol. Microbiol. 28 (3) (1998) 593-601.

[35] M. Christmann, B. Kaina, Nuclear translocation of mismatch repair proteins MSH2 and MSH6 as a response of cells to alkylating agents, J. Biol. Chem. 275 (46) (2000) 36256-36262.

[36] G.A. Belov, C. Habbersett, D. Franco, E. Ehrenfeld, Activation of cellular Arf GTPases by poliovirus protein 3 CD correlates with virus replication, J. Virol. 81 (17) (2007) 9259-9267.

[37] M.K. Morphew, 3D Immunolocalization with plastic sections, in: J.R. McIntosh (Ed.), Methods in Cell Biology, 79, Academic Press, 2007, pp. 493-513.

[38] R. Vidya Priyadarsini, R. Senthil Murugan, S. Maitreyi, K. Ramalingam, D. Karunagaran, S. Nagini, The flavonoid quercetin induces cell cycle arrest and mitochondria-mediated apoptosis in human cervical cancer (HeLa) cells through p53 induction and NF-KB inhibition, Eur. J. Pharmacol. 649 (1-3) (2010) 84-91

[39] E.G. Gamaly, A.V. Rode, B. Luther-Davies, Ultrafast ablation with high-pulserate lasers. Part I: Theoretical considerations, J. Appl. Phys. 85 (8) (1999) 4213-4221.

[40] A. Tavangar, B. Tan, K. Venkatakrishnan, Study of the formation of 3-D titania nanofibrous structure by $\mathrm{MHz}$ femtosecond laser in ambient air, J. Appl. Phys. 113 (2) (2013) 023102.

[41] G. Odachi, R. Sakamoto, K. Hara, T. Yagi, Effect of air on debris formation in femtosecond laser ablation of crystalline Si, Appl. Surf. Sci. 282 (2013) 525-530.

[42] K. Vasilev, Z. Poh, K. Kant, J. Chan, A. Michelmore, D. Losic, Tailoring the surface functionalities of titania nanotube arrays, Biomaterials 31 (3) (2010) 532-540.

[43] A. Tavangar, B. Tan, K. Venkatakrishnan, Synthesis of bio-functionalized threedimensional titania nanofibrous structures using femtosecond laser ablation, Acta Biomater. 7 (6) (2011) 2726-2732.

[44] J.Y. Lim, M.C. Shaughnessy, Z. Zhou, H. Noh, E.A. Vogler, H.J. Donahue, Surface energy effects on osteoblast spatial growth and mineralization, Biomaterials 29 (12) (2008) 1776-1784.

[45] H.-A. Pan, Y.-C. Hung, Y.-P. Sui, G.S. Huang, Topographic control of the growth and function of cardiomyoblast H9c2 cells using nanodot arrays, Biomaterials
33 (1) (2012) 20-28.

[46] J.B. Recknor, D.S. Sakaguchi, S.K. Mallapragada, Directed growth and selective differentiation of neural progenitor cells on micropatterned polymer substrates, Biomaterials 27 (22) (2006) 4098-4108.

[47] J.Y. Park, M. Morgan, A.N. Sachs, J. Samorezov, R. Teller, Y. Shen, K.J. Pienta, S. Takayama, Single cell trapping in larger microwells capable of supporting cell spreading and proliferation, Microfluid. Nanofluid. 8 (2) (2009) 263-268.

[48] F. Notta, S. Doulatov, E. Laurenti, A. Poeppl, I. Jurisica, J.E. Dick, Isolation of single human hematopoietic stem cells capable of long-term multilineage engraftment, Science 333 (6039) (2011) 218-221.

[49] N. Faucheux, R. Schweiss, K. Lützow, C. Werner, T. Groth, Self-assembled monolayers with different terminating groups as model substrates for cell adhesion studies, Biomaterials 25 (14) (2004) 2721-2730.

[50] E. Delivopoulos, M.M. Ouberai, P.D. Coffey, M.J. Swann, K.M. Shakesheff, M. E. Welland, Serum protein layers on parylene-C and silicon oxide: effect on cell adhesion, Colloids Surf. B Biointerfaces 126 (2015) 169-177.

[51] L.T. Allen, M. Tosetto, I.S. Miller, D.P. O'Connor, S.C. Penney, I. Lynch, A. K. Keenan, S.R. Pennington, K.A. Dawson, W.M. Gallagher, Surface-induced changes in protein adsorption and implications for cellular phenotypic responses to surface interaction, Biomaterials 27 (16) (2006) 3096-3108.

[52] J. Wei, T. Igarashi, N. Okumori, T. Igarashi, T. Maetani, B. Liu, M. Yoshinari, Influence of surface wettability on competitive protein adsorption and initial attachment of osteoblasts, Biomed. Mater. 4 (4) (2009) 045002.

[53] G.B. Sigal, M. Mrksich, G.M. Whitesides, Effect of surface wettability on the adsorption of proteins and detergents, J. Am. Chem. Soc. 120 (14) (1998) 3464-3473.

[54] P. Ying, Y. Yu, G. Jin, Z. Tao, Competitive protein adsorption studied with atomic force microscopy and imaging ellipsometry, Colloids Surf. B Biointerfaces 32 (1) (2003) $1-10$.

[55] A.-S. Andersson, F. Bäckhed, A. von Euler, A. Richter-Dahlfors, D. Sutherland, B. Kasemo, Nanoscale features influence epithelial cell morphology and cytokine production, Biomaterials 24 (20) (2003) 3427-3436.

[56] W.C. Clem, S. Chowdhury, S.A. Catledge, J.J. Weimer, F.M. Shaikh, K. M. Hennessy, V.V. Konovalov, M.R. Hill, A. Waterfeld, S.L. Bellis, et al., Mesenchymal stem cell interaction with ultra-smooth nanostructured diamond for wear-resistant orthopaedic implants, Biomaterials 29 (24) (2008) 3461-3468.

[57] V. Chin, B.E. Collins, M.J. Sailor, S.N. Bhatia, Compatibility of primary hepatocytes with oxidized nanoporous silicon, Adv. Mater. 13 (24) (2001) 1877.

[58] J. Wei, M. Yoshinari, S. Takemoto, M. Hattori, E. Kawada, B. Liu, Y. Oda, Adhesion of mouse fibroblasts on hexamethyldisiloxane surfaces with wide range of wettability, J. Biomed. Mater. Res. B Appl. Biomater. 81B (1) (2007) $66-75$. 